\title{
BTT \& RFLB - THE OPTIMUM SET FOR STEAM TURBINE BLADES MONITORING
}

\begin{abstract}
Miroslav BALDA
Research and Testing Institute, Tylova 46, 30100 Plzeñ, Czech Republic

correspondence: balda@vzuplzen.cz

ABstract. BTT, Blade Tip Timing system, is a commercially available system generating files of precise times of blade tips when passing sensors attached in a machine stator. RFLB, Residual Fatigue Life of Blades, is a postprocessor of those files evaluating estimates of fatigue lives of all blades fitted to the wheel. The set reduces a danger of unexpected blade failures.
\end{abstract}

KEYwords: BTT, RFLB, fatigue, blades, maintenance.

\section{INTRODUCTION}

Fatigue of materials is a very complicated phenomenon, which influences the quality of structures seriously. The bigger the structure is, the more important are the consequences of possible fatigue damages. The typical structures, in which any fatigue cracks should not occur, are turbomachines. Any unexpected breakdown caused by the fatigue generates extreme losses of energy production. This is the reason why manufacturers of turbomachinery devote enormous care to a design of fatigue-save parts of a machine and to material testing. To common tests belong also fatigue tests of most exposed parts of the machine, blades of a turbine.

The blades are working at rather complicated operational conditions like high radial stress from centrifugal forces and extreme velocity of wet steam turbulent flow exciting them to vibrations accompanied by alternating stresses. Dynamic stresses at critical points of blades may initiate material damage, which results in a crack propagation when cumulated. Since this phenomenon may end in a total breakdown of a blade followed by an unplanned shutdown of the machine, producers of turbomachines arm their products with systems, which are able to monitor blades at their critical places.

\section{Blade Tip Timing - BTT}

The current monitoring systems used for that purpose are denoted as BTT - Blade Tip Timing systems because they are based on time measurement of blade tips when passing sensors (see Fig. 1).

A BTT system consists of a set of sensors placed at the stator of the turbine above tips of blades of the selected wheel. Outputs of $n_{s}$ sensors are connected to control inputs of their own counters of precise clock pulses. As soon as a tip of the vibrating blade passes a sensor, the sensor generates a pulse which initiates a transfer of the current content of the counter to its memory. There is still one sensor and the counter and its memory which counts pulses generated by a datum fixed at a shaft. At the end of the measurement, the contents of all memories are sent to $n_{s}+1$ files. The measured data, series of times, are processed offline into natural frequencies of blades and histograms of position magnitudes and other parameters of the measurement.

\section{Residual Fatigue Life of BLADES - RFLB}

An application of a system RFLB has in principle three main stages:

- measurement of material properties,

- modal analysis of the blade to be measured,

- long time monitoring in situ in a power plant.

All stages are very important for the final statement about the residual fatigue life of all measured blades.

\subsection{MATERIAL PROPERTIES}

There are two material tests necessary for RFLB system, the tensile test, and the high cycle fatigue test. The result of the tensile test is shown in the form of the diagram made by a testing device in Fig.2 It may be accepted as a limiting case of the fatigue test during which the specimen has been broken at the half of the loading cycle.

The high cycle fatigue (HCF) test is a different one. Usually, a set of 8 - 10 specimens are loaded by harmonic processes of different amplitudes $\sigma_{a m}$ up to breaks after $N_{a m}$ loading cycles [1]. The result of the test is a regression line to couples $\left(N_{a m}, \sigma_{a m}\right)$, called SN curve [2, which becomes a straight line when drawn in a diagram with axes $\log N_{a m}, \log \sigma_{a m}$ (see Fig.3).

\subsection{MODAL ANALYSIS}

Good information on the blade as an object of monitoring is very important. The detailed numerical analysis of the blade fitted in the wheel is made by a FEM program. Results of the analysis should contain the solution of the eigenvalue problem, e.g. natural 


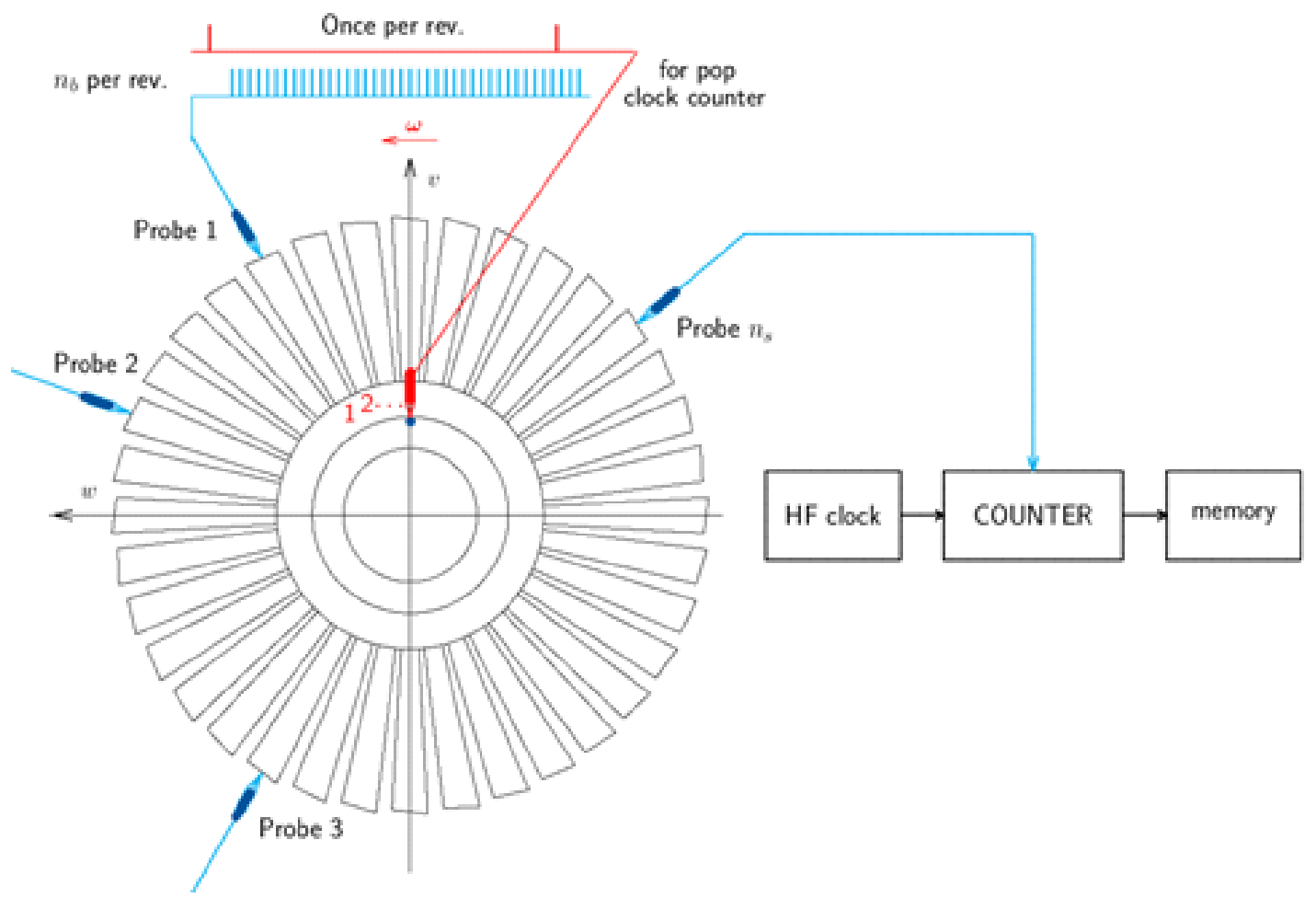

FiguRE 1. BTT system with $n_{s}$ probes (sensors).

frequencies and corresponding natural modes for a set of nodal diameter numbers. Moreover, it is also important to have frequency response functions of circumferential deflection of a blade tip to stress tensors at critical places of the blade.

\subsection{Monitoring By the RFLB}

The software system RFLB complements the system BTT by estimating fatigue lives of blades. It exploits the information contained in the BTT output files for the purpose totally.

Such a complicated system, as the RFLB is, should be split into modules fulfilling special tasks. The interactive MATLAB program RFLB is built as a flexible one as possible with respect to events occurring in power plants. The system contains about 30 scripts and functions.

The control module - RFLB organizes, in a cooperation with the operator, the function of the system RFLB. At first, the operator informs the module where to find the parameters of the blade, material, and results from the modal analysis. The operator may also interrupt the normal automatic run of the RFLB when needed. There are many reasons for doing so. A typical situation occurs when the operator wants to look into the protocol or wants to plot the result of some past measurement. Then, the program breaks the normal sequence of commands, fulfills the requirement of the operator, and continues just in the place, where has been interrupted. This behavior ensures the control module of the program. The module RFLB initiates the run of the RFLB with all new blades. It also enables to change some blades without a loss of continuity in the cumulation of damages of other blades. The same holds after a break in processing.

The module BTT is a driver for a continuous run of the system. It distributes tasks to specialized functions. The first of them is function getdata which reads the files generated by systems BTT. There are served files from two BTT systems, Starman and Hood. The function getdata is the only one which should be rebuilt if another BTT system was applied for measurements. The reason for it is that the output files of systems BTT are not standardized.

The main role of function getdata is creating a vector composed of key-phasor times of once per revolution pulses from the red probe in Figure 1 and a hyper-matrix (3D array) of times from all sensors and blade tips of the wheel. One vector of the 3D array is seen from Figure 4. Since the measured data are in the standard form, so the rest of the RFLB system does not need any change. At the end of the function getdata, all-time series are Fourier transformed into aliased Fourier spectra by fast Nieslony's function rainflow [3]. The resulting Fourier spectra are aliased in frequency due to the deep under-sampling of blade tip signals.

Frequency aliasing has to be eliminated by a spectrum reconstruction. This is implemented by function reconstr. It is well known that under-sampled signals defy reconstruction by normal way. Fortunately, there is additional information at disposal. That is a set of natural frequencies obtained during the numerical 


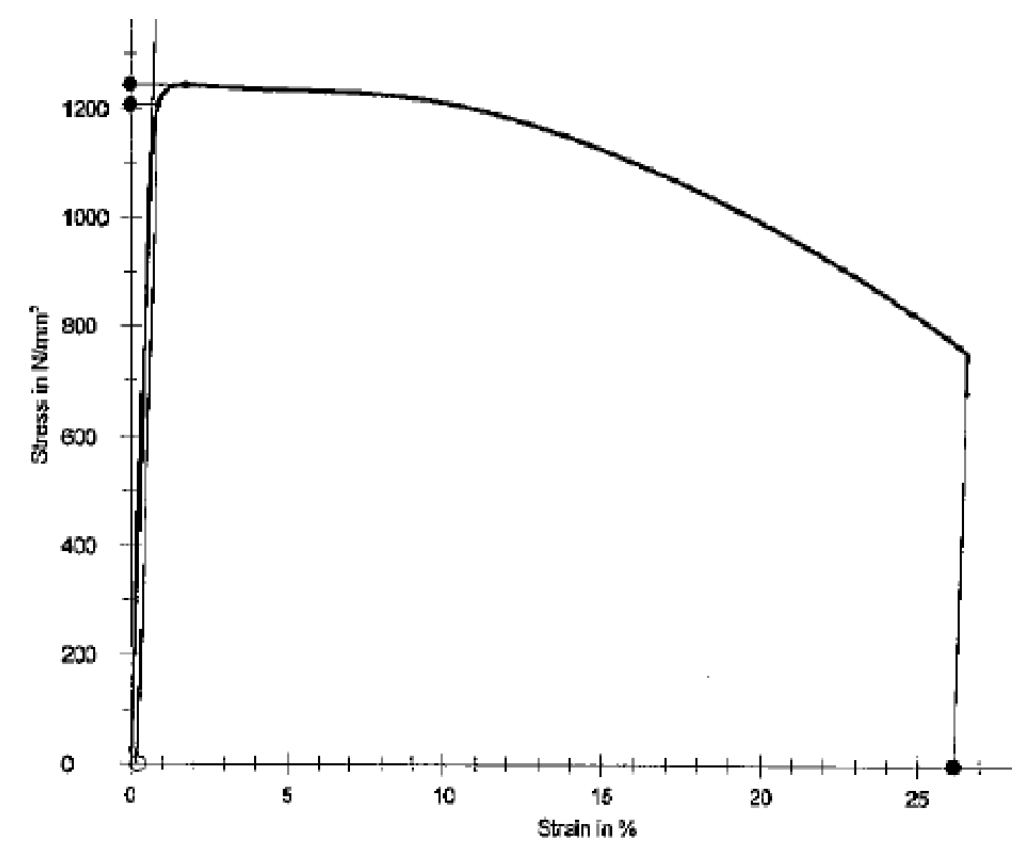

Figure 2. Material T671 - tensile test.

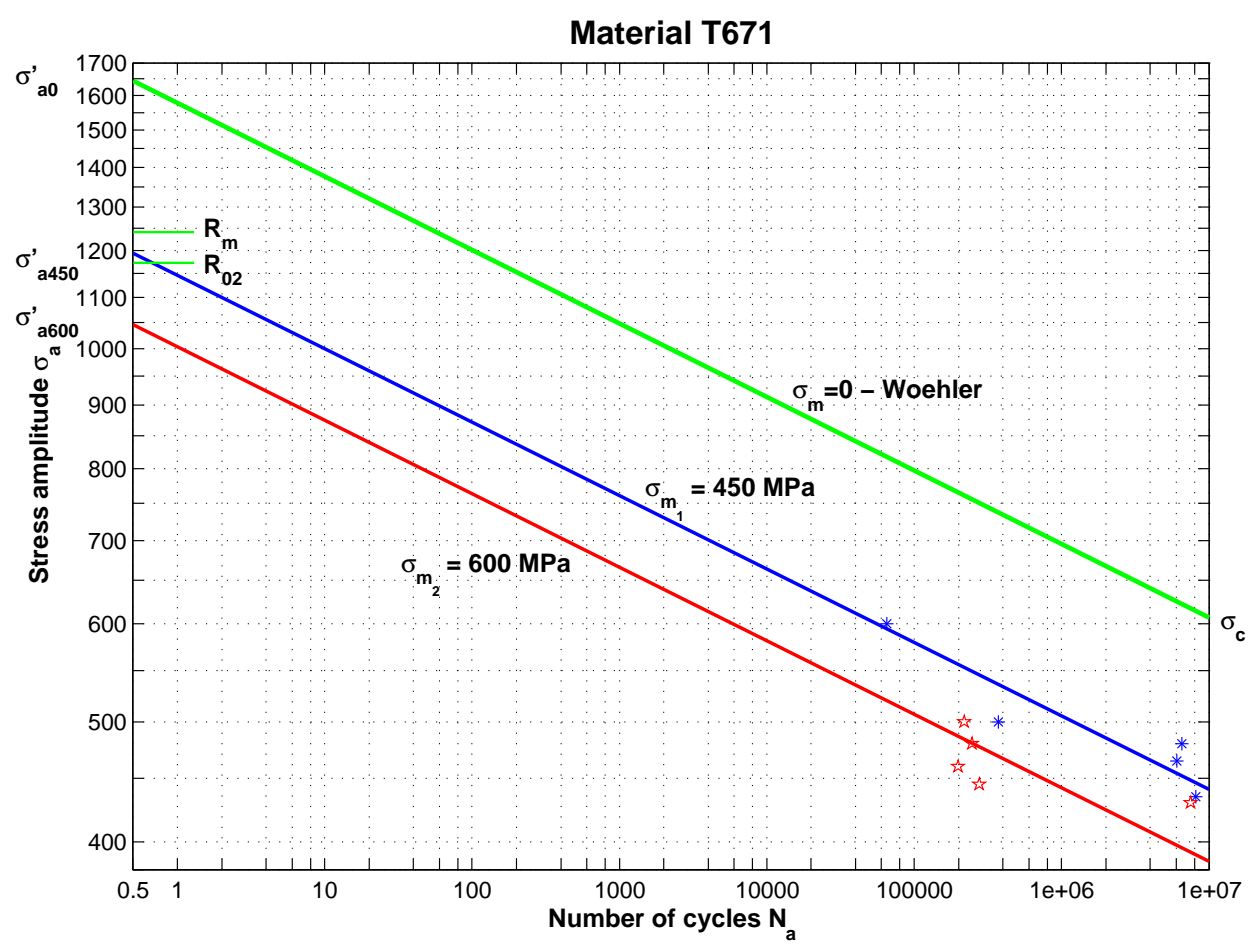

Figure 3. T671 SN curves, $\sigma_{m}=0,450$, and $600 \mathrm{MPa}$.

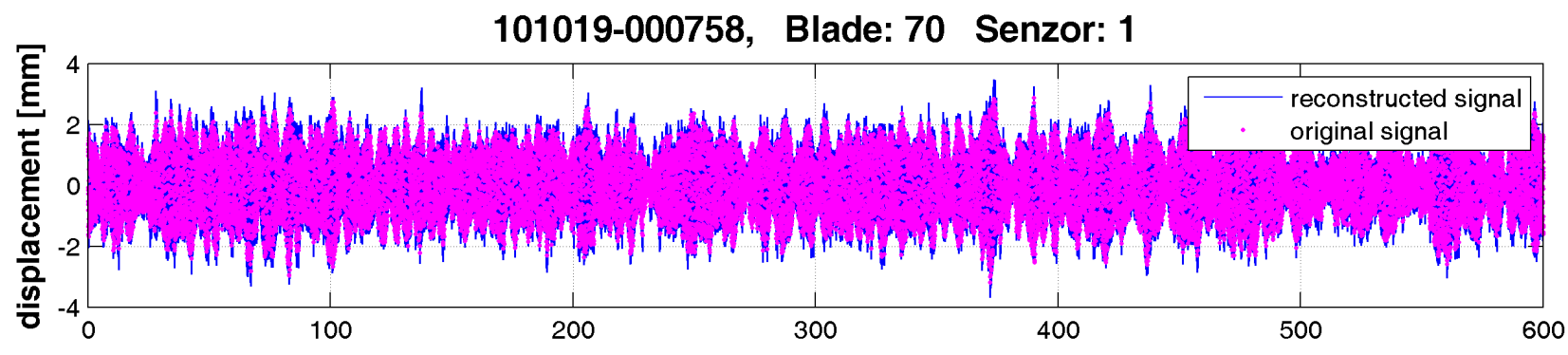

FIGURE 4. Vector of measured and reconstructed samples of one blade tip deflections. 


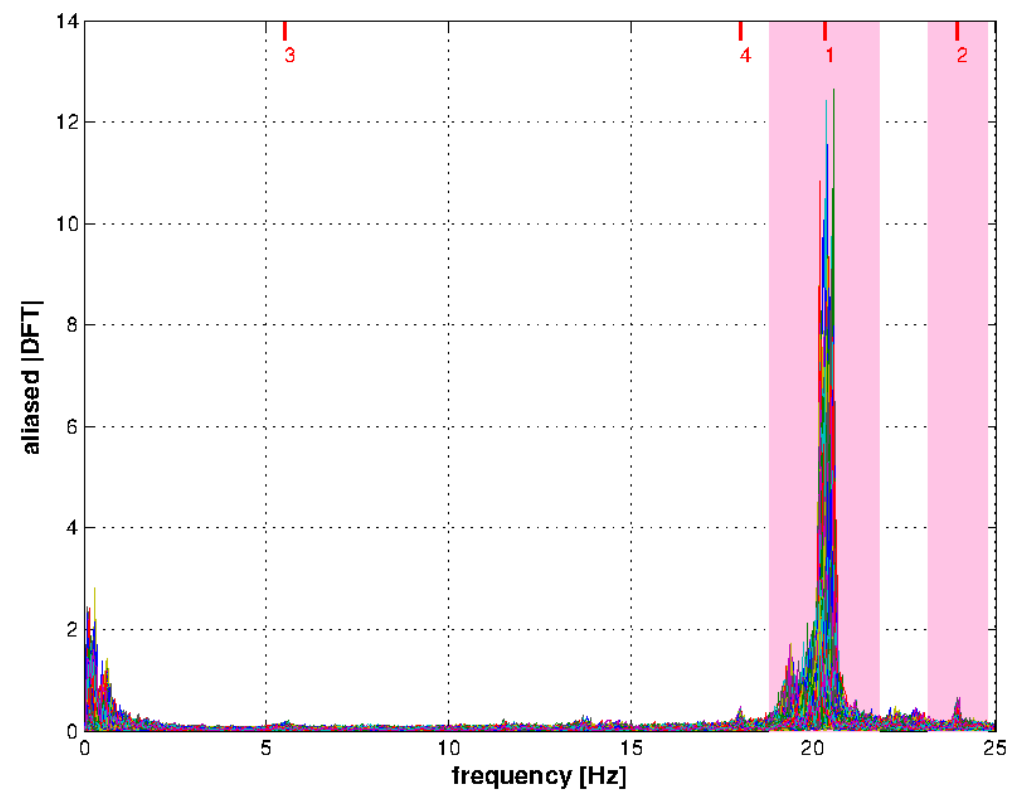

FiguRE 5. Aliased DFT of all blades in the wheel.

modal analysis. The natural frequencies recalculated to the aliased ones serve for identification of evaluated peaks in DFT. The frequency bands around DFT peaks coinciding with aliased natural frequencies are moved on places corresponding real natural frequencies of the reconstructed spectra.

In Figure 5 there are positions of aliased natural frequencies shown in upper parts of the diagram by red ticks and order numbers. The upper frequency of the reconstructed Fourier spectra is chosen by an order higher than the speed frequency, which is the sampling frequency $f_{s}$, say, $f_{S}=25 f_{s}$. The rest of the aliased spectra is spread within the reconstructed spectra under user wishes. The last stage of the reconstruction is the inverse Fourier transform of the reconstructed spectra, what returns reconstructed signals in much higher resolution as seen in Figure 4 If the moving of the spectrum bands was done with care, the reconstruction is successful, what is seen from Figure 4 .

As soon as the reconstructed signals are ready, relative damages in critical places of the blades can be estimated by function damage. Unfortunately, it is the most time-consuming operation, because of each blade signal has to undergo rain-flow analysis, what is a decomposition of each signal into full cycles, followed by a recalculation of time data into circumferential deviations and then to stress state in critical places on blades.

For the purpose, the elements of the stress tensors obtained during modal analysis, are used for evaluating an effective (damaging) stress $\sigma_{d}$ of $k$ th loading cycle in the following form

$$
\begin{aligned}
\tilde{\sigma}_{d, k, n} & =\Delta t_{k} \times\left[\tilde{\sigma}_{x}^{2}+\tilde{\sigma}_{y}^{2}+\tilde{\sigma}_{z}^{2}\right. \\
& -2 \mu\left(\tilde{\sigma}_{x} \tilde{\sigma}_{y}+\tilde{\sigma}_{y} \tilde{\sigma}_{z}+\tilde{\sigma}_{z} \tilde{\sigma}_{x}\right) \\
& \left.+k_{c}^{2}\left(\tilde{\tau}_{x y}^{2}+\tilde{\tau}_{y z}^{2}+\tilde{\tau}_{z x}^{2}\right)\right]_{n}^{1 / 2},
\end{aligned}
$$

Blade damages up to time 120628-021449

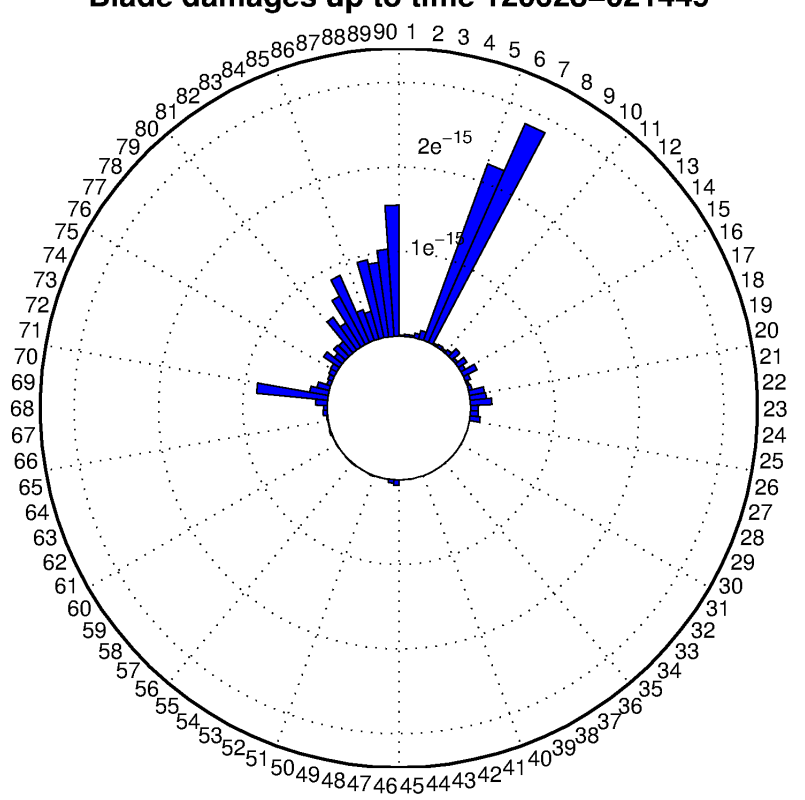

Figure 6. Relative damages of all blades.

where $k_{c}=\sigma_{c} / \tau_{c} \approx \sqrt{2(1+\mu)}$ and symbols with tilde are dynamic components of a stress tensor in the critical point corresponding to $n$th mode of vibration. Time-series of damaging stress course are decomposed into a set of full cycles and half cycles by a rainflow procedure getting thus stress amplitudes $\sigma_{a k}$ and mean stresses $\sigma_{m k}$ needed for an estimation of elementary damage $d_{k}$ in $k$ th cycle by a fatigue hypothesis. The most common one is the hypothesis of PålmgrenMiner [4, 5] respecting an influence of $\sigma_{m}$ [6]:

$$
d_{k, \mathrm{PM}}=1 / N_{a m}=2\left(\frac{s_{a}}{s_{a 0}^{\prime}-s_{m}}\right)^{w},
$$

where fictive amplitude $s_{a m}^{\prime}$ replaces either $\sigma_{a m}^{\prime}$ or 
$\tau_{a m}^{\prime}$. It is evident from Figure 3 that its values are given by the positions of intersections of a particular SN curve with the line $N_{a m}=0.5$. For all family of SN curves holds that $s_{a m}^{\prime}=s_{a 0}^{\prime}-s_{m}$. This property of SN curves of steels saves time and money because it canceled the necessity of making expensive tests of prestressed materials.

The system RFLB cumulates elementary relative damages defined by equation (2), so it is able to yield information on the total relative damage $D \leq 1$ of any blade of the wheel to the operator at any moment. In case, the damage $D$ reached a chosen amount, the system issues an alarm message. Residual fatigue life, defined as $L=1-D$, says how much time still remains to break down.

\section{Conclusions}

The article has shown the advantages gained by a combination of a hardware system BTT with the software system RFLB. Installation of the set of both items in power plants for monitoring blades in potentially uncertain turbine stages may be very effective for the reliability of a machine because it diminishes a danger of unexpected breakdowns and enables to plan times of maintenance and a number of spare blades in store.

\section{LIST OF SYMBOLS}

$d_{a m}$ relative damage of a cycle with $\sigma_{a}$ and $\sigma_{m}$

$D$ total relative damage

$f_{n}$ natural frequency $[\mathrm{Hz}]$

$f_{s} \quad$ sampling frequency $[\mathrm{Hz}]$

$f_{S} \quad$ sampling frequency of reconstructed spectra $[\mathrm{Hz}]$

$f_{N} \quad$ Nyquist frequency $=f_{s} / 2[\mathrm{~Hz}]$

$L$ relative residual fatigue life

$N_{a m}$ number of cycles to failure under $\sigma_{a}$ and $\sigma_{m}$

$R_{m}$ material strength [MPa]
$R_{p}$ yield limit $[\mathrm{MPa}]$

$s$ either $\sigma$ or $\tau[\mathrm{MPa}]$

$t$ time [s]

$T_{s} \quad$ sampling period $=1 / f_{s} \quad[\mathrm{~s}]$

$w$ exponent of Woehler's curve

$\mu$ Poisson's ratio

$\sigma$ stress in tension [MPa]

$\sigma_{a}$ amplitude of a stress cycle $[\mathrm{MPa}]$

$\sigma_{m}$ mean stress of a stress cycle [MPa]

$\sigma_{a m}$ amplitude of a cycle by mean stress $[\mathrm{MPa}]$

$\sigma_{a m}^{\prime}$ intersection point of SN curve with $N=0.5[\mathrm{MPa}]$

$\tau$ stress in torsion [Mpa]

\section{ACKNOWLEDGEMENTS}

The author appreciates a kind support of his research from the Technological Agency of The Czech Republic via the Center of Competence No. TE01020068, Center of research and experimental development of reliable energy production

\section{REFERENCES}

[1] J. Chvojan: A test of material T671 high cycle fatigue (In Czech), VZÚ Plzeň, Testing record No. 846/2003

[2] ČSN 42 0368: Fatigue testing of metals. Statistical evaluation of fatigue test results of metals (In Czech), Czech Normalizing Institute, Prague, 1973.

[3] A. Nieslony: Rainflow Counting Algorithm. MathWorks, File Exchange, file No. 3026, 2003

[4] A. Pålmgren: Die Lebensdauer von Kugellagern, VDI Zeitschrift (68), pp. 339-341, 1924.

[5] M.A. Miner: Cumulative damage in fatigue, Trans. ASME, Journal of Applied Mechanics, (12) pp. A159-A164, 1945.

[6] M. Balda: Fatigue life assessment of prestressed material, Engineering Mechanics, C. Fischer \& J. Náprstek eds., ITAM CAS CZ, Proc. Conf. Engineering Mechanics, Svratka, May 14-17, 2018 